\title{
Analytical Performance of a Fiber Optic Probe Dissolution System
}

\author{
Kevin Bynum,1* Kurt Roinestad1, Abe Kassis1, J ohn Pocreva2, \\ Lane Gehrlein 1, Frank Cheng1, Philip Palermo1 \\ 1 Purdue Pharma LP, International Research and Development Center, Ardsley, NY \\ 2 Emisphere Technologies Inc., Tarrytown,NY
}

\author{
email: kevin.bynum@pharma.com \\ *to whom correspondence should beaddressed
}

\begin{abstract}
AUV Fiber Optic Probe Dissolution System has been developed for theanalysis of solid dosage forms. The system uses 12 dip-typefiber-optic probes coupled to 12 separate Photo DiodeArray (PDA) spectrophotometers to acquire continuous dissolution curves in real time. The system is applicableto theanalysis of both immediate and controlled-release formulations. Thesystem is accurate, quicker, and easier to set up when compared with conventional HPLC or UV-sipper systems. The data is acquired, calculated, and secured using an in-house developed and validated software package. The software package usesa novel scatter-correction algorithm to generateaccurate dissolution curves in a turbid medium. Thedata that the system generates is asaccurateas the HPLCmethods that it is replacing.
\end{abstract}

Introduction

issolution testing is a time-consuming and labor-intensive test procedure.The technique requires a sample of dissolution medium be removed from each of the test vessels at a specified time point(s). This sampling procedure can be handled either manually or by means of an automated sipper mechanism. Each method has its own inherent errors associated with it. The manual technique is subject to analyst variability. Two of the most common errors associated with the manual sampling technique are improper sampling within the vessel and timing errors in sampling. Additionally, this is the most labor-intensive technique and is especially taxing for controlled-release formulations, which often require 24-hour testing periods. Automatic sampling techniquescan save analyst labor, but still require a good deal of maintenance and set-up time.Also, automated sipping mechanisms are complexand contain many moving parts that can malfunction, resulting in sampling errors.Sipper mechanisms are prone to sample contamination, carryover, bubbles, dilution, filter clogging, and tubing leaks.

An In-Situ dissolution technique will eliminate all of these sampling problems. A number of researchers have investigated this technique to study the feasibility of this technology(1,2,3). More recently work has been done with probes in traditional sampling location $(4,5,6,7)$, as well as with a probe-in-shaft sampling technique(9).

This publication will discuss a fiber optic probe dissolution system that we have developed at Purdue Pharma LP.What sets our device apart is that it is a fully integrated system consisting of validated hardware and software. We have sucessfuly used the system in our R\&D laboratory as well as our quality control facility.

\section{System Description}

TheUV fiber optic dissolution system utilizes a diptype UV probe to measure the amount of active drug released from a solid dosage form. A total of 12 UV transflectance probes are placed into 12 dissolution vessels and reside insitu for the duration of the test. Each probe is connected to a miniature Photo Diode Array (PDA) spectrometer via a fiber optic light guide.A pair of deuterium lampsare used asillumination sources.A UV spectrum is then acquired from each of the vessels at set intervals. The raw spectral data is stored in a database on our secured computer network, and the resultant dissolution point is displayed in real time. The data control computer, using our validated proprietary software package RAINBOW performsall necessary calculations. The system contains no moving parts (Figure 1).

To add flexibility to our system we used a probe

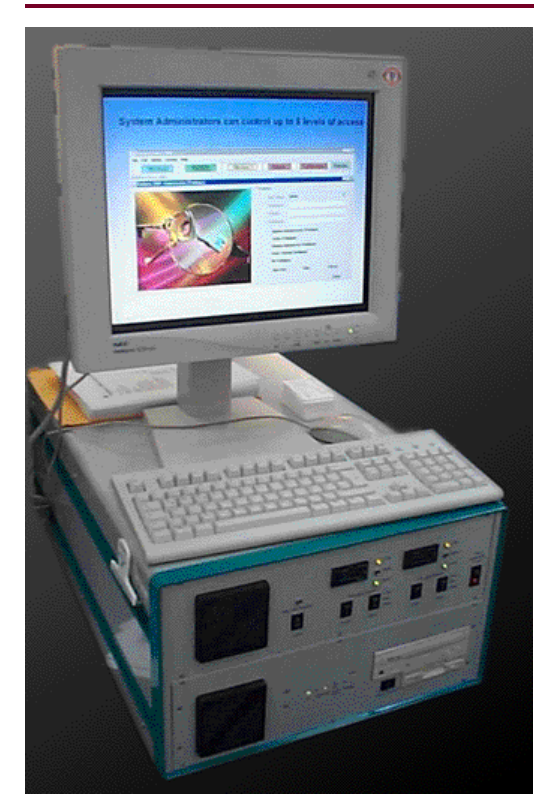

Figure 1: UV Fiber Optic Probe Dissolution System

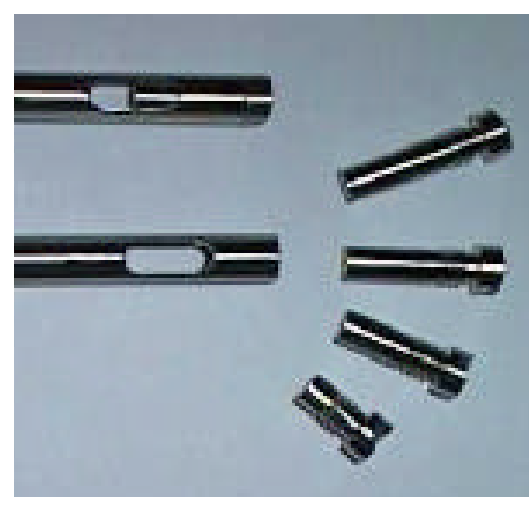

Figure 2: Assorted Tips with a removable flow cell; a wide range of pathlengths may be used with the system (Figure2). We are currently using probes with $2 \mathrm{~cm}$ to $0.2 \mathrm{~cm}$ 


\section{Fiber Optic Probe .... continued}

removable pathlength tips (Hellma Cells, Forrest Hills,NY). These probeshave been shown to provide excellent analytical performance with the added flexibility provided by the interchangeable tip.

The system utilizes twelve Photo-Diode Array (PDA) spectrometers. We use 12 Zeiss MMSUV detectors (Hellma Cells, Forrest Hills, NY). This allows for the acquisition of a full UV spectrum $(200 \mathrm{~nm}-404 \mathrm{~nm})$ from all twelve vessels in less than 10 seconds. The system is typically configured to sample every ten minutes for $12-24$ hours in the case of a controlled release formulation, or every 30 seconds for an immediate release formulation. Faster sampling times are realized if fewer vessels are sampled. If a single probe is used the scan rate can approach one scan per second.

The system can be interfaced into any standard dissolution apparatus. We have used the system successfully with the Hanson SR8, Hanson SR6,Distek Premiere 5100 and the VanKel 7010 dissolution apparatus.

The system currently uses an in-house developed and validated software package, RAINBOW, to collect, store, and calculate dissolution curves in real time. This package has been fully validated for use in a GMP environment. The system stores all data in a Microsoft Access database located on our secured server.All data is audit trailed, and a multitiered security system assures the integrity of the data collected with the system.

The multi-tiered security system allows restricted access to certain functions in the software. Only method development chemists can write and modify methods.QC chemists can then load a locked, read only, method, and start dissolution. Everyone has the ability to view data that has been collected with the system, but no one can delete any data.Once a dissolution is collected, it is automatically archived. RAINBOW has no delete button.

Additionally, the system has an energy normalization feature that automatically sets all of the energy levels on each of the 12 detectors to an optimal operating level.This is a critical feature that makes this sort of multi-channel analysis possible.

The software has a comprehensive system suitability feature that assures that the analyst cannot start a dissolution unless a number of rigorous conditions are met. The system first collects absorbance spectra from two separate standard solutions. Two suitability calculationsare then performed, the \% difference between the two standard solutions, and the \%RSD of the 5 scans of the second standard solution. These results are calculated for each probe, and the results for each probe must be less than the specified limit in order to begin the dissolution procedure.If the $\%$ RSD or $\%$ difference is greater than the specified limit for any probe then the experiment is aborted, and the analyst must correct the problem and repeat the system suitability procedure. This ensures that there are no configuration problems with the system (lamp not lit, lamp energy low, bubble in probe) before the dissolution is started.

The software also contains calculation methods to correct for scattering interference. Two types of scattering correction can be applied. A baseline correction can be applied to correct for minor scattering interference, or a second derivative can be ap plied to correct for more severe interference. All of these calculations are performed in real time as the scans are taken.

Finally, the software has a number of integrated calibration methods to ensure instrument performance.The system has an integ rated wavelength calibration routine that uses a primary standard (low pressure mercury lamp) to check for wavelength accuracy.Additionally, photometric accuracy (linearity and reproducibility) are checked with a NIST traceable optical standard, obtained from APS analytical standards.

\section{Methods and Materials}

\section{Materials}

Commercially available $325 \mathrm{mg}$ Bufferin tablets (Lot \#904518) were purchased for the profile accuracy study.Aspirin reference standard was purchased from Sigma Aldrich,St LouisMO (lot \# 67H9421). USP reference standard Caffeine (lot I) wasused for the stability study and the linearity study as well as the scattering correction study.A NIST traceable photometric accuracy standard (lot \#16yys) was also used for the scattering correction study, and was obtained from APS Analytical Standards Inc. (Redwood City, CA).

\section{Equipment}

Four different types of dissolution apparatus were utilized for this study. Hanson SR6 and SR8 dissolution apparatus were obtained from Hanson Research (Chatsworth, CA). A Vankel 7010 with dipping manifold was obtained from VanKel (Cary, NC). A Distek Premier 5100 bathless dissolution apparatus was obtained from Distek (North Brunswick,NJ).

A number of different Type II UV Fiber Optic Probe dissolution systems were used to collect the data in this study. Each consisted of 12 MMSUV Photo Diode Array (PDA) spectrometers, 12 dip type probes with removable flow cells (Hellma 
Cells, Forrest Hills, NY), 2 deuterium lamps (Oriel Instruments, Stratford,Ct), 2 fiber optic splitters (Hellma Cells, Forrest Hills, NY), and a dual Pentium II 233MHz computer workstation with 128 Mbytes of ram,8Gbyte hard drive (Appro International Inc,Milpitas, CA).

\section{Methods}

See Table 1 for a listing of the parameters that were used for each dissolution test conducted.

\section{Experimental}

For all experiments, the system was set up by first acquiring dark, $100 \%$ transmittance, and standard measurements. This allowed for the accurate generation of absorbance spectra.

\section{IR Profile Accuracy}

A commercially available Aspirin formulation was used to test the accuracy of the system. The fiber optic unit was configured to calculate the amount of drug released by subtracting the baseline measurement, $350 \mathrm{~nm}$, from the peak measurement at $260 \mathrm{~nm}$. The single tab let was dropped, and the vessel was sampled manually numerous times throughout the dissolution of the tablet. The samples were then filtered through a 0.45 micron filter, and the amount of drug measured by mean s of an off-line UV assay.

\section{Control Release Accuracy}

The accuracy of our system with a controlled release formulation was studied on an in-house, controlled release, analgesic formulation. In this study, 12 samples were analyzed by acquiring UV spectra every ten minutes for 24 hours. Once the tablets were dropped at the start of the run,the system acquired, stored, and calculated all of the results without further analyst intervention. The dissolution profile was generated by subtracting the baseline absorbance at $304 \mathrm{~nm}$ from the peak absorbance at $272 \mathrm{~nm}$. The results of this experiment were compared to a separate experiment that was conducted using the HPLC method that the fiber optic system has replaced.

\section{Precision Study}

The intermediate precision of the technique was assessed by having a number of analyststest the same formulation, on different days. Three separate fiber optic dissolution systems were used for this study.This is the same formulation that was used in the controlled release accuracy study.

\section{Linearity Study}

The linear range of the system was studied using caffeine as a model compound.

The system was configured with a $20 \mathrm{~mm}$ pathlength probetip. The probe was then placed in a beaker containing $900 \mathrm{ml}$ of deionized water, and a series of aliquots of a caffeine solution were added to the beaker.A full UV scan wastaken following the addition of each aliquot.This range simulates a $10 \mathrm{mg}$ caffeine formulation dissolving in $900 \mathrm{ml}$ of water. The lowest concentration studied represents the $0.1 \%$ release level (10 micrograms in $900 \mathrm{ml}$ of buffer) of the simulated formulation.

\section{Long-term Stability}

The long-term stability of the system was studied by analyzing a constant amount of drug (caffeine) over a twelve hour period. The system was set up to take a scan every 10 minutes for 12 hours. A Hanson SR-8 dissolution system was set up with six vessels filled with $900 \mathrm{ml}$ of deionized water.The UV Probe system was then configured by acquiring dark, $100 \%$ transmittance and standard scans. Once the system had passed the suitability criteria,the 12 hour acquisition began. Each of the six vessels was spiked with a small amount of caffeine solution. The system was allowed to acquire scans for 12 hours.

\section{Scattering Correction}

The accuracy of our proprietary, second derivative based, scattering correction algorithm was studied in this experiment.After configuring the system to use just a sing le point baseline correction, a single dissolution vessel was spiked with a small amount of caffeine and allowed to acquirea single data point of this pure solution. Immediately following this scan, an aliquot of turbidity standard was introduced into the vessel, to simulate turbidity

Table 1. Parameters for Dissolution Test.

\begin{tabular}{|lccccc|}
\hline Sample & Apparatus & $\begin{array}{c}\text { Vessel } \\
\text { Volume }\end{array}$ & Speed & Media & Pathlength \\
\hline $\begin{array}{l}\text { Bufferin } \\
\text { (Lot \#904518) }\end{array}$ & Paddle & $500 \mathrm{ml}$ & $75 \mathrm{rpm}$ & $\begin{array}{c}0.05 \mathrm{M} \text { Acetate Buffer } \\
\text { (pH } 4.50 \pm 0.05)\end{array}$ & $0.2 \mathrm{~cm}$ \\
\hline $\begin{array}{l}\text { 24hour control } \\
\text { release formulation }\end{array}$ & Paddle & $900 \mathrm{ml}$ & $100 \mathrm{rpm}$ & $\begin{array}{c}900 \mathrm{~mL} \text { of phosphate } \\
\text { buffer (pH } 6.50 \pm 0.05)\end{array}$ & $1.0 \mathrm{~cm}$ \\
\hline
\end{tabular}




\section{Fiber Optic Probe ... continued}

arising from the disintegration of a solid dosage form. The system was allowed to collect two scans of this solution. A second and third aliquot of turbidity standard was added to the vessel in the same manner. Following the dissolution the results were recalculated from the raw absorbance data using the second derivative algo rithm, and the results compared.

\section{Results}

\section{IR Profile Accuracy}

The results of the profile accuracy study are illustrated in Figure 3.This experiment demonstrates the rapid scanning ability of the system. The system is able to acquire a dissolution data point, on all 12 channels, once every 15 seconds. If a single probe is used the scan rate can approach one scan per second. In this experiment the system was configured to acquire a dissolution point at 30 second intervals. This rate was sufficient to provide a very detailed profile of the release from this immediate release formulation. The manual samples that were withdrawn from the vessel during the dissolution, and assayed with an off-line UV method, are consistent with the fiber optic results. This demonstrates the accuracy of this method throughout the entire experiment for a typical disintegrating tablet.

\section{Controlled Release Accuracy}

Figure 4 illustrates the ability of the system to acquire data accurately over an extended period of time, in this case 24 hours. Once the system is set up, and passes system suitability (requires about 30 minutes), the system will run without analyst intervention for the remainder of the dissolution. The comparison data for this experiment is from the existing HPLC method that the fiber optic method is replacing. The HPLC data was acquired by a different analyst on a different set of tablets. It should be noted that there is no hydrodynamic off set between the HPLC data (collected with nonresident sampling probes) and the UV Probe data (collected with resident fiber optic probes).

\section{Precision Study}

The results of the precision study shows the performance of the system in a "real world" environment. The results of five different analysts, running the same lot of tablets on three different systems is illustrated in Figure 5. For each experiment the average of 12 tablets is presented. The tight correlation of all five data sets demonstrates the degree of analytical repeatability that can be realized with a spectroscopic system, which has no moving parts.

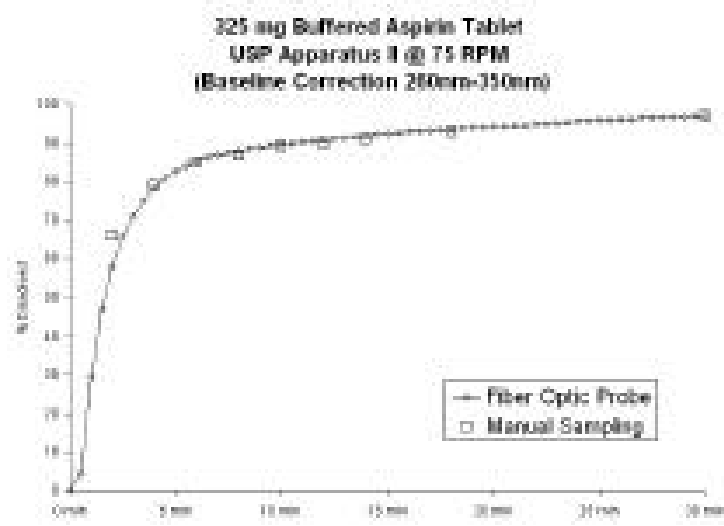

Figure 3: IR Profile Accuracy

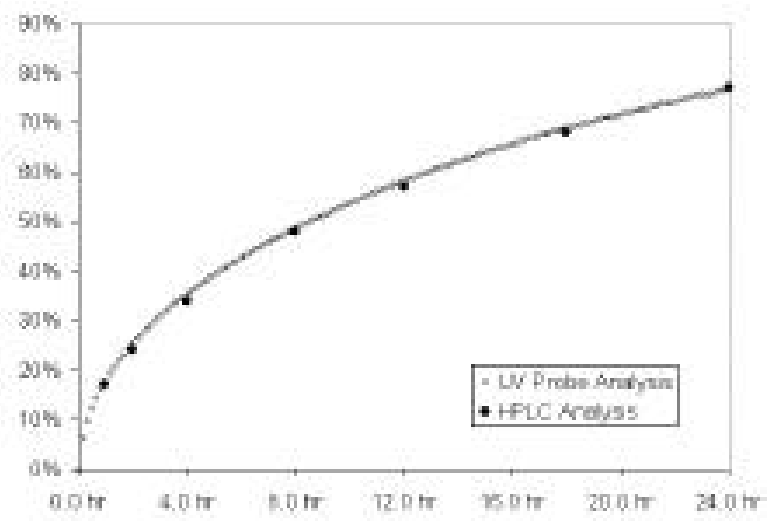

Figure 4: Controlled release formulation

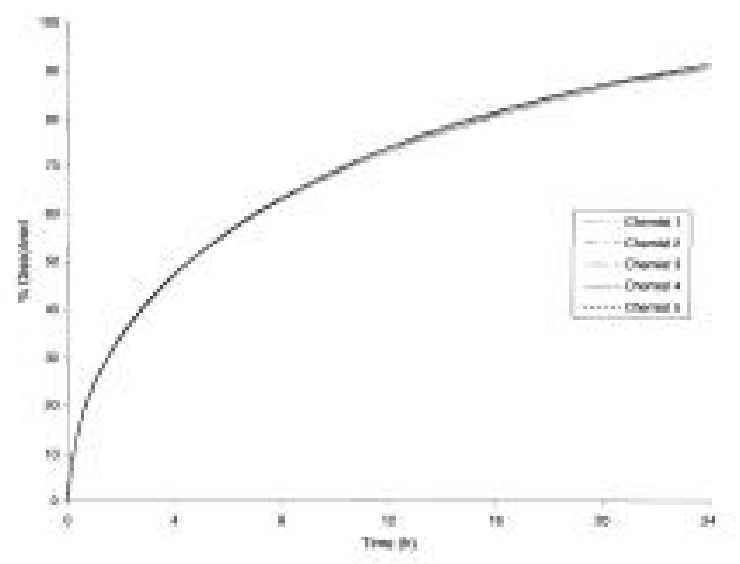

Figure 5: Precision study, compiled from 5 dissolutions 


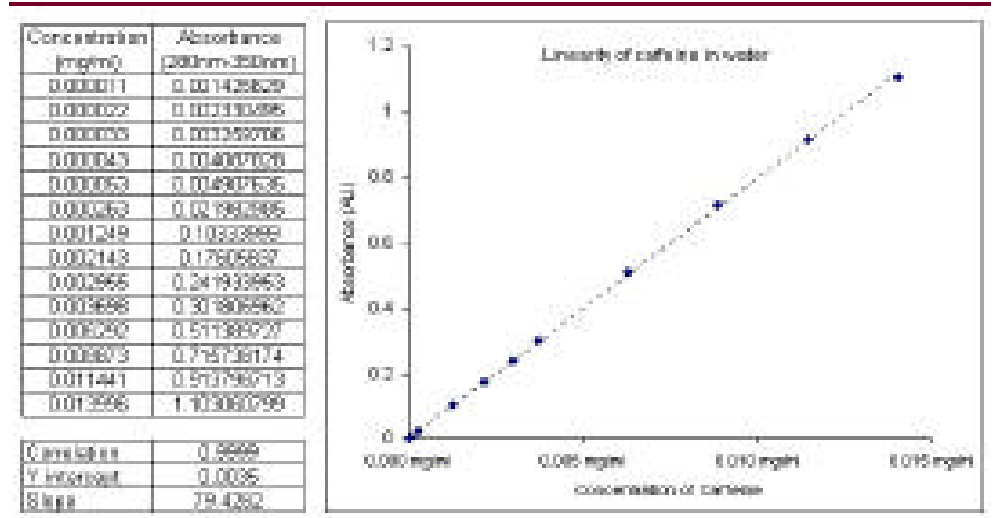

Figure 6: Linearity Study.

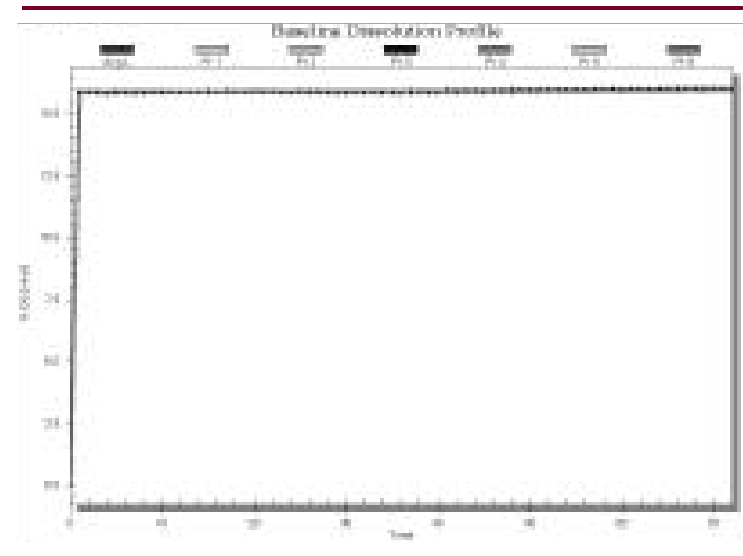

Figure7:12 hour system stability

\section{Linearity Study}

The results of the linearity study are presented in Figure 6. These results demonstrate that the system can be used to measure very low levels of drug. Higher levels of drug can be just as easily measured by changing the path length of the tip, to reduce the amount of absorbance from the sample.

\section{Long-term Stability}

The stability of the system is demonstrated in Figure 7.The constant \%dissolved measurement over 12 hours, on six individual probes, demonstrates the ability of the system to make accurate analytical measurements during a controlled-release dissolution. This level of system stability is possible due to the design of the system. The system uses no moving parts so there is very little variability over time. Each channel has an independent Photo Diode Array so no switching or multiplexing is needed. The choice of a high quality deuterium lamp and Photo Diode Array provide the extremely stable environment necessary to make these continuous measurements.

\section{Scattering Correction}

The system's ability to correct for scattering is demonstrated in Figures 8 and 9. These figuresshow how the system is able to make constant analytical measurements in an environment where an increasing level of interference is present. This experiment was orig inally run with the simple baseline correction method, and is shown in Figure 8.The baseline method shows a significant off-set as the concentration of turbidity standard increases in the vessel. Figure 9 shows the same data, recalculated with the second derivative method. The constant amount of caffeine present in the vessel is accurately measured, even in the increasing environment of the scattering interference.

\section{Discussion}

The system has proven to not only save considerable analysis time, but also improve the quality of the dissolution data acquired. Additionally, the system's integrated quality assurance features streamlines regulatory compliance.

A user will typically set up the UV fiber optic probe dissolution system in 2 hours. This includes all media preparation, degassing, and equilibration.Once the dissolution experiment has started no further analyst intervention is needed. As UV scans are collected, the data is time stamped and stored on our secured network. Results are then calculated, displayed, and secured in real time. Once the dissolution is completed, the analyst only has to press the "print report" button to obtain a hard copy of the dissolution results. All results are stored in the database automatically, so no post dissolution paperwork is needed.

The system suffers from very little "down time"since there are no moving parts.

The system has proven to be as accurate, and in some cases more accurate, when compared to on-line UV and HPLC methods. The technique completely eliminates all errors associated with the removal of sample from the vessels. The errors which typically plague the dissolution chemist include sample carryover, leaking/clogging of transfer lines, pump failures, clogging of filters, and absorption of drug onto filters/tubing. With these errors removed, the accuracy and precision of the system is limited by the spectroscopic technique that is employed to make the analytical measurement.

The single largest obstacle to an accurate in-situ UV absorbance measurement is interference from excipients. Molecular absorption from pharmaceutical excipients is rarely a problem if spectral measurements are made at wavelengths longer than $240 \mathrm{~nm}$. The UV measurements that are made with this system focus on the $\pi^{*}$ transition that typically occurs between 250 and $300 \mathrm{~nm}$. Since most pharmaceutical active ingredients have an aromatic system,this region is very useful for quantitative measurements. By focusing in on this region, we gain a good deal of specificity, since almost all pharmaceutical excipients do not have significant UV absorption in this spectral region. This provides 


\section{Fiber Optic Probe ... continued}

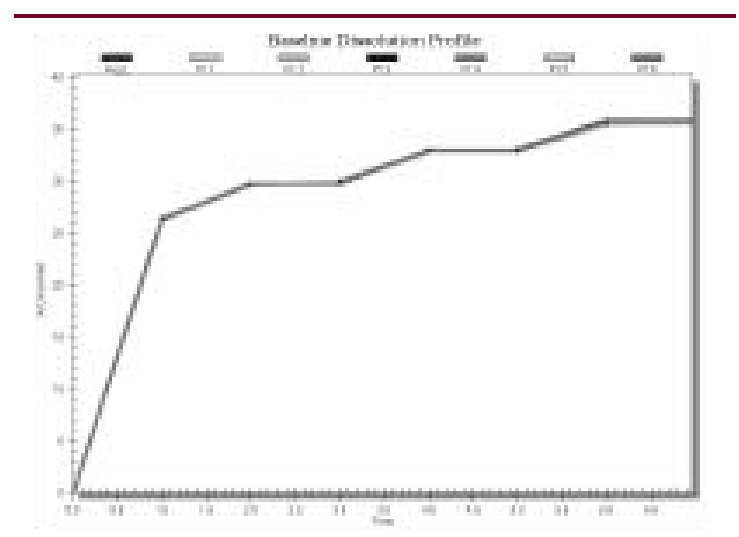

Figure 8: Constant amount of caffeine using baseline correction

us with a "spectral window" above $240 \mathrm{~nm}$ where we have specificity for our compound of interest.

Scattering by pharmaceutical excipients is a much greater interference that is typically present at all wavelengths across the UV spectrum. This scattering interference is usually present at one of two levels. The first is a relatively mild scattering that is caused by the film coating of non-disintegrating pharmaceutical dosage forms. This form of scattering is evident as a slight (0.01-0.10 AU) baseline off-set. This type of scattering is corrected by our baseline correction algorithm, which is the default setting of our system. This al gorithm simply subtracts the baseline absorbance at a given wavelength from the peak absorbance measurement (Figure 10). We have found that sort of correction works well with non-disintegrating, and even some disintegrating formulations.

The second level of scattering interference is much more severe. This type of scattering produces a sloping baseline, where the interference becomes greater at shorter wavelengths. This sort of scattering is known as Tyndall scattering (named after British physicist J ohn Tyndall [1820-1893]), and is observed in colloidal suspensions. This sort of scattering arises from the particulate in a disintegrating formulation, or from gelatin capsules. Gelatin capsules exhibit a rather severe level of scattering as shown in Figure 11. Not only is there a baseline offset, but the baseline slopes upward from longer to shorter wavelengths. Additionally, the degree of interference can be variable in intensity, as shown by the difference in the baseline for all three samples in Figure 11. This makes it nearly impossible to remove such an interference by simple subtraction a placebo spectrum.

The solution to the interferance caused by scattering is to remove the sloping baseline. The easiest way to do this is to take a second derivative of

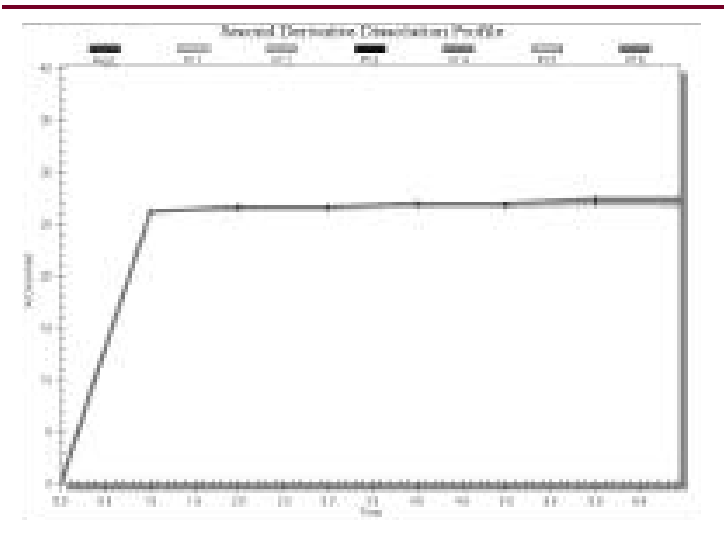

Figure 9: Constant amount of caffeine using second derivative calculation

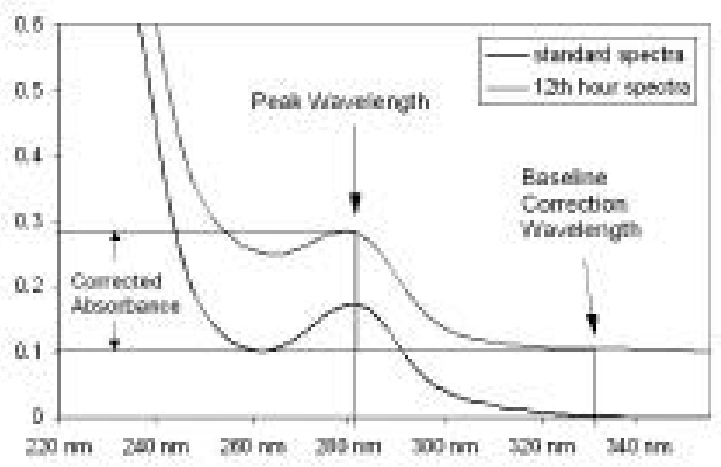

Figure 10: Baseline correction method

each spectrum. This will serve to remove any sloping off-set, since the second derivative of a sloping line is zero. An example of this is shown in Figure 12 , which shows two absorbance scans from the scattering correction experiment shown in Figures 8 and 9. The top of the figure shows the absorbance of the pure standard, and the same amount of standard with a scattering interference (turbidity standard). The turbid solution demonstrates a typical turbid interference, with a sloping baseline. The correction for this interference is illustrated in the bottom of the figure. As is clearly evident, the second derivative removes the baseline off-set and slope of the turbid interference. The second derivative spectrum of the turbid solution is almost identical to that of the pure standard. In the wavelength range of $275-305 \mathrm{~nm}$, an almost perfect scattering correction has occurred.

The precision of the method is likewise significantly enhanced, even more than the accuracy, when compared to non in-situ dissolution methods. This is because there is very little instrumentto-instrument variability. The most critical parameter at the start of a run is the amount of energy 


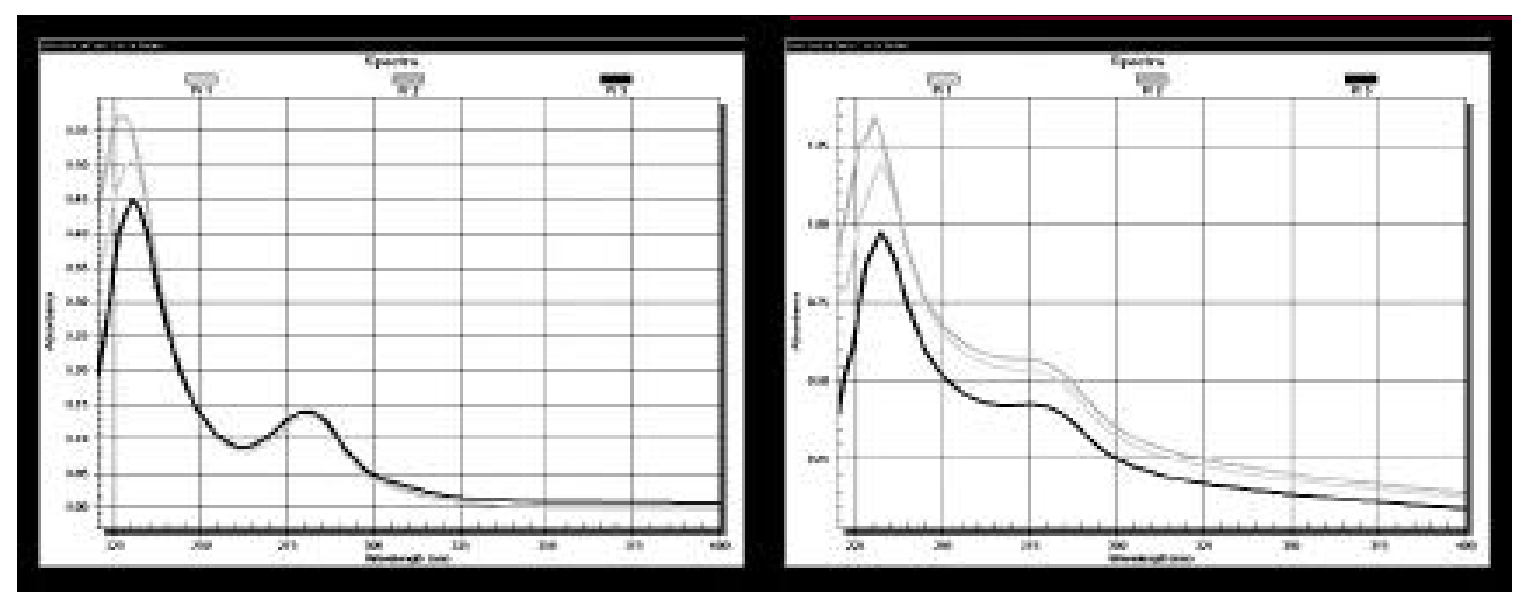

Figure 11: Gelatin capsule interference: Standard spectra (left) without sloping baseline and 24-hour dissolution sample spectra (right) with sloping baseline interference.

that is reaching the detectors, before the tablets are dropped. Since this parameter is tightly controlled by the energy normalization feature and system suitability procedure, we can make sure that each system is operating in an analytically sound manner prior to the start of a dissolution. Additionally, the fact that there are no moving parts in the system means that any sort of mechanical failure is highly unlikely.

Finally, the choice of spectrometers makes this system a solid analytical platform. The Zeiss MMS spectrometer exhibits excellent analytical performance in the absorbance range of $0.02 \mathrm{Au}$ up to $1.50 \mathrm{Au}$. The titanium body of the Zeiss spectrometer provides exceptional wavelength accuracy. We have observed less than a $0.1 \mathrm{~nm}$ annual drift. This stable detection unit allows us to reproducibly measure a dissolution profile very accurately. This fact is clearly illustrated in Figure 5, where five separate analysts ran the same lot of tablets on three different instruments, with almost identical results.

This method is not just a quicker way of conducting a dissolution test. It opens many new doors for the R\&D chemist. The high rate of sampling allows for a much higher data density. Since we use an independent spectrometer for each channel, we can sample from all 12 channels at rates of once every 15 seconds. A single probe can sample as fast as once a second. This will result in dissolution profiles that contain hundreds of points. This high rate of sampling will also allow for the profiling of explosive release compounds. This will also make any statistical analysis on a dissolution data set much more rugged and strengthen any in-vivo/in-vitro correlation significantly. Additionally, this data density may allow for the prediction of dissolution curves.
The problems associated with studying nanoparticle formulations will also be removed by this method, since there is no sampling of the solution. Finally, the acquisition of a full spectrum at every time point allows the R\&D chemist to view a dissolution in a whole new manner. A "dissolution surface" can be observed by plotting all of the UV spectral data vs.time on a 3D coordinate system. One such example from the author's laboratory is shown in Figure 13. This is a very efficient way of demonstrating spectral purity of the sample over a given period of time.

Finally, a more subtle, but increasingly important advantage of this system is the compliance features associated with a well designed data system. The system automatically collects all data and stores it on the users validated computer network without any analyst intervention. All results and user actions are time stamped to demonstrate data integrity. All data is available for viewing after a dissolution experiment, but cannot be deleted by the analyst. All of the calculations (fully validated) are safely locked away in the software, and cannot be changed. This completely eliminates the many errors associated with post experiment manual calculations. This saves not only the time of the analyst conducting the experiment, but also the time of the supervisor who must check the calculations.

\section{Conclusion}

A UV Fiber Optic Probe Dissolution System has been developed for the analysis of solid dosage forms. The system uses 12 dip-type fiber-optic probes coupled to 12 separate PDA spectrophotometers to acquire continuous dissolution curves in real time. The system is applicable to the analysis of both immediate and controlled-release for- 


\section{Fiber Optic Probe ... continued}
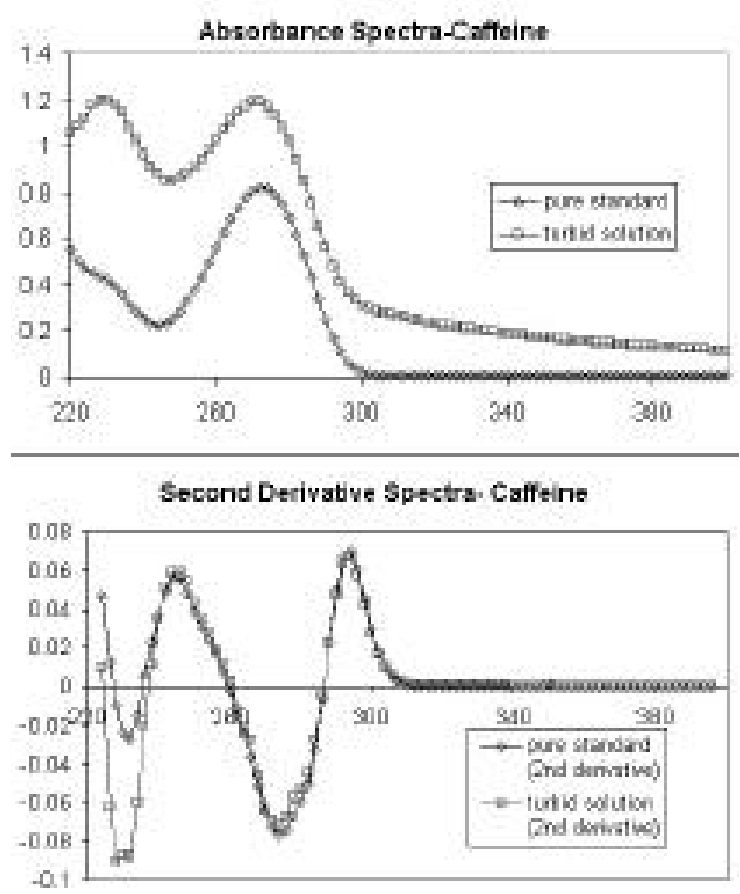

Figure 12: Correcting for scattering with the 2 nd derivative calculation

mulations. The system is accurate, quicker, and easier to set up when compared with conventional HPLC or UV-sipper systems. The data is acquired, calculated, and secured using an in-house developed and validated software package. The software package uses a novel scatter-correction algorithm to generate accurate dissolution curves in a turbid medium. The data that the system generates is as accurate as the HPLC methods that it is replacing.

\section{REFERENCES}

1.J.H.Cho, P.J. Gemperline, D. S. Walker, Wavelength Calibration Method for a CCD Detector and Multichannel Fiber-Optic Probes, Applied Spectroscopy volume 49 No 12,(1995)

2. . .H. Cho, P. . Gemperline, A. Salt, and D.S. Walker, UV/Visible Spectral Dissolution Monitoring by in Situ Fiber-Optic Probes, Anal Chem, volume 67, No 17, (1995)

3. Aldridge P.K., Kostek L.J ., In Situ Fiber Optic Dissolution Analysis, Dissolution Technologies, volume 2, issue4, 10-11 (1995)

4.I.Nir, B.J ohnson, J.J ohansson,C.Schatz, Application of Fiber-Optic Dissolution Testing for Actual Products, Pharmaceutical Technology, Volume 25, NO 5, 2001

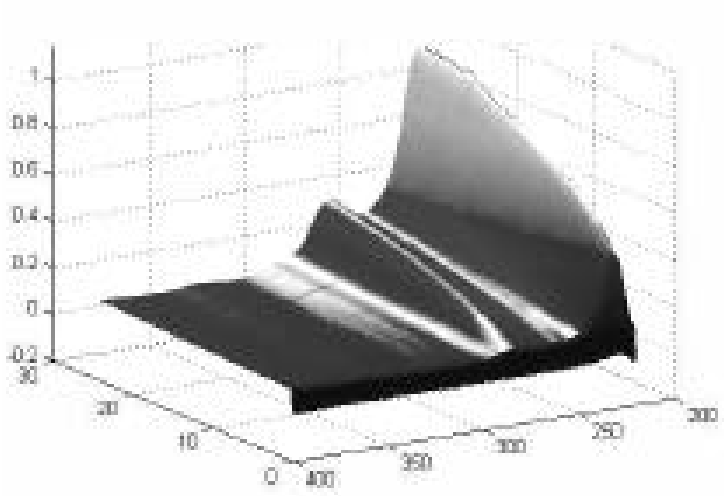

Figure 13: 3D "dissolution surface" of a controlled release (24 hour) formulation

5.K.C. Bynum, E. Kraft, A New Technique in Dissolution Testing, Pharmaceutical Technology, volume 23 NO 10 (1999)

6.K.C. Bynum, E. Kraft, J.Pocreva, E.W. Ciurczak, P. Palermo, In Situ Dissolution Testing Using a Fiber Optic Probe Dissolution System, Dissolution Technologies, volume 6, Issue 4, (1999)

7.J . Earnhardt, I.Nir, Fiberoptic Dissolution Testing Advances Drug Quality Control, Spectroscopy, Volume 15 No 2 (2000)

8.C.Schatz,M .Ulmschneider, R. Altermatt, S. Marrer, Reader's response: Hollow shaft Sampling With Fiber Optics, Dissolution Technologies, volume 7 issuel,(2000)

9. C Schatz,M.Ulmschneider, R. Altermatt, S. Marrer, H. Altorfer, Testing fibre optic immersion probes for UV/vis spectroscopy, Spectroscopy Europe, volume 12, NO 6,2000

10. Basic Chemistry, William S. Seese, Guido H. Daub, 3rd Edition, Prentice Hall Inc

Send correspondence to:

Kevin Bynum Purdue Pharma LP International Research and Development Center 444 Sawmill River Rd.

Ardsley, NY 10502 